\title{
Home source for cryo-EM
}

\section{Dimple Karia ${ }^{1}$, Adrian Koh ${ }^{1}$, Zuzana Hlavenkova ${ }^{2}$, Milos Malinsky², Vojtech Dolezal ${ }^{2}$, Alevtyna Yakushevska ${ }^{1}$, Lingbo $\mathrm{Yu}^{1}$, Abhay Kotecha ${ }^{1}$}

\author{
${ }^{1}$ Thermo Fisher Scientific, Materials and Structural Analysis Division, Achtseweg Noord, Eindhoven, Netherlands \\ ${ }^{2}$ Thermo Fisher Scientific Brno s.r.o., Materials \& Structural Analysis, Brno, Czech Republic \\ dimple.karia@thermofisher.com
}

Single Particle Analysis (SPA) application of cryo-electron microscopy (cryo-EM) has become one of the dominating methods for 3D structure determination of a wide variety of biological macromolecules to understand their function, mechanism of action ${ }^{[1]}$ and protein ligand/drug interactions. However, as the popularity of this technique increases, so does the need for accessibility and improved efficiency. In this abstract, we describe two cryo-Transmission Electron Microscopes (cryo-TEMs), that are equivalent to home source X-ray diffractometers, but for cryo-EM.

The first is the Thermo Scientific Tundra cryo-TEM operating at $100 \mathrm{kV}$ with a semi-automated grid loading system and automated data collection for SPA. Tundra allows users to load the sample in an effortless and robust way. Using this new microscope, we solved structures of several soluble and membrane protein samples. Standard sample such as apoferritin protein (equivalent to lysozyme crystals for X-ray crystallography) was solved to $2.6 \AA$ resolution. More challenging samples such as homo-pentameric human $\mathrm{GABA}_{\mathrm{A}}$ (gamma-aminobutyric acid type A) receptor was resolved to $3.4 \AA$ reconstruction. The GABA $\mathrm{A}_{\mathrm{A}}$ receptor is a small membrane protein and ligand-gated chloride-ion channel that mediates inhibitory neurotransmission. $\mathrm{GABA}_{\mathrm{A}}$ receptors are important therapeutic drug targets and hence it is vital to understand the molecular mechanism by which these receptors mediate neurotransmission. After decades of efforts, in 2014, this same sample of $\mathrm{GABA}_{\mathrm{A}}$ receptor was crystallized and structure resolved to $3.0 \AA^{[2]}$. With cryo-EM on Tundra, we obtained similar resolution without the need of crystallization and in near native conditions.

To further push for more automation and high-throughput, we used the Thermo Scientific Glacios ${ }^{\mathrm{TM}}$ cryo-TEM. Glacios has an Autoloader $^{\mathrm{TM}}$, with a robotic arm which can load 12 grids simultaneously and switch the grids automatically. To push for higher resolution, Glacios is also equipped with direct electron detector (DED) and can be combined with Selectris energy filter. Using this system, we achieved a $2.4 \AA$ resolution cryo-EM map for the same $\mathrm{GABA}_{\mathrm{A}}$ receptor. Both these microscopes are not only good for sample screening and optimization but are also capable for generating high resolution structures comparable to those obtained from Xray crystallography experiments.
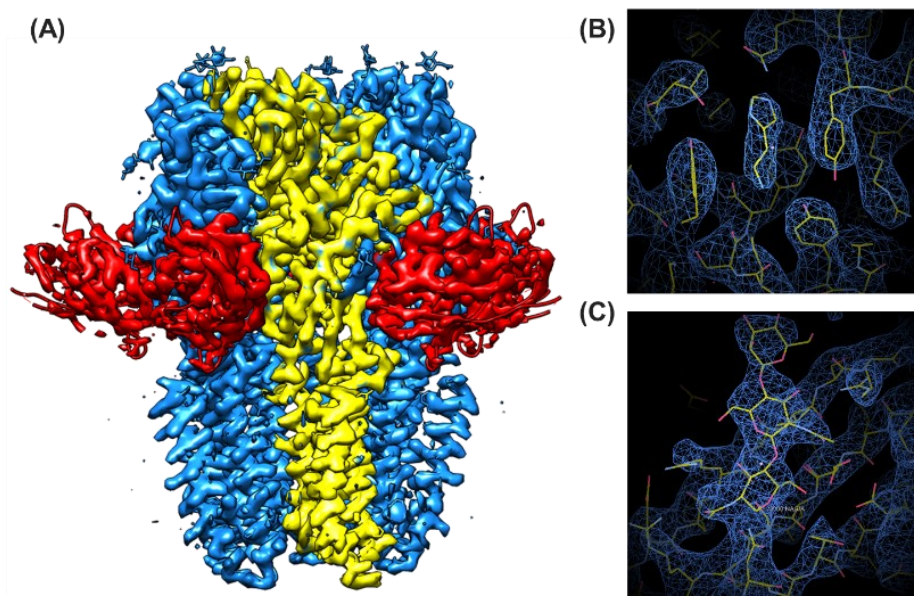

Figure 1. A, 3.4A reconstruction of $\mathrm{GABA}_{\mathrm{A}}$ receptor from Tundra. B, ligand density and $\mathrm{C}$, sugars.

[1] Michael Eisenstein. The field that came in from the cold. Nature, Vol.13 No.1, (2016).

[2] Miller, P., Aricescu, A. Crystal structure of a human GABAA receptor. Nature 512, 270-275 (2014).

Keywords: cryo-EM, Single Particle Analysis, Tundra, Glacios, GABAA receptor 